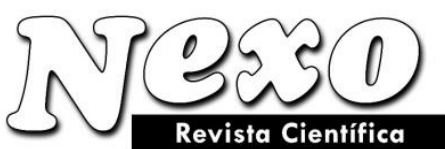

Vol. 36, No. 06, pp. 1632-1648/Diciembre 2021

\title{
Calidad microbiológica y fisicoquímica del agua en cuatro lagos cráter del estado Puebla, México
}

\section{Microbiological and physicochemical quality of water in four crater lakes in the state of Puebla, Mexico}

\author{
Juan Ricardo Cruz-Aviña ${ }^{1,2}$, Laura G. Núñez-García ${ }^{3}$, Rubén Cabrera ${ }^{4 *}$, Jhoana Díaz-Larrea ${ }^{3}$,
} Elsa I. Castañeda-Roldan ${ }^{2}$.

${ }^{1}$ Universidad Tecnológica Calakmul (UTC), Departamento de Recursos Naturales, Carr Xpujil-

Dzibalchen, Km. 2+260, Xpujil, Calakmul, CP 24640, Campeche, México.

${ }^{2}$ Benemérita Universidad Autónoma de Puebla, Ciudad Universitaria, Av. San Claudio s/n, Col San Manuel, CP.72592, Puebla, México.

${ }^{3}$ Universidad Autónoma Metropolitana unidad Iztapalapa. División de Ciencias Biológicas y de la Salud, Departamento de Hidrobiología, Av. San Rafael Atlixco 186, Col. Vicentina, Ciudad de México, 09340.

México.

${ }^{4}$ Gabinete de Arqueología, Oficina del Historiador de la Ciudad, Habana Vieja, Cuba. Calle Tacón \# 12 e/ O’Reilly y Empedrado, Ciudad de La Habana, Cuba.

* cabreraalgas@gmail.com

(recibido/received: 02-octubre-2021; aceptado/accepted: 15-noviembre-2021)

\section{RESUMEN}

Se realizó la calidad microbiológica del agua (CMA) de cuatro lagos cráter, Alchichica (ALCHI), Atexcac (ATx), La Preciosa (LPr) y Quechulac (QUE), de manera mensual durante un año. Se utilizaron protocolos para estimar coliformes totales (CT), coliformes fecales (CF), enterococos (ENT), E. coli, (E.coli +Rodococcus) y Clostridium perfringers. Se detectó a E. coli. en QUE, el más contaminado, seguido por ALCHI y LPr. El pH, OD y temperatura no variaron en el estudio. La salinidad fluctuó de 0 a $10.0 \mathrm{mgL}^{-1}$. Utilizando la asociación con Rodococcus se estableció que ATx, LPr y ALCHI presentaron contaminación derivada del ganado, mientras QUE presentó contaminación fecal de origen humano. E. coli no subsiste en los Axalapascos, por lo que es alóctono al sistema y oportunista por las descargas fecales del ganado circundante debido a malas prácticas agropecuarias. Los niveles de contaminación microbiológica en los lagos cráter, superan la normatividad vigente para uso humano, agropecuario, de turismo.

Palabras claves: Contaminación microbiológica; E. coli; lagos Maars.

\section{ABSTRACT}

Authors carried out the QMW analysis, on four crater Alchichica (ALCHI), Atexcac (ATx), La Preciosa (LPr) and Quechulac (QUE), with monthly frequency during one year. Protocols were used to evaluate total coliforms (TC), fecal coliforms (FC), enterococcus (ENT), E. coli, (E. coli + Rodococcus) and Clostridium 
perfringers. The presence of $E$. coli was detected in all the studied lakes, and the most contaminated was QUE, followed by ALCHI y LPr. The $\mathrm{pH}$, DO and the temperature no recorded variation. The salinity registered fluctuations 0 a $10.0 \mathrm{mgL}^{-1}$. ATx, LPr and ALCHI recorded contamination derived from goats, while QUE registered fecal contamination from human source. The presence of $E$. coli is variable in Axalapascos, because of that is allochthones to the system and opportunistic due to the surrounding cattle fecal discharge associated to unwell agricultural practice. The levels of microbiological contamination in the crater lakes, exceeding regulations for the human, agricultural and tourism.

Keywords: Microbiological contamination, E. coli, Marss lakes.

\section{INTRODUCCIÓN}

Evaluar la calidad microbiológica (CMA) de un cuerpo de agua epicontinental resulta de gran importancia, dado el riesgo asociado con la ingesta de agua contaminada por microorganismos provenientes de material fecal (Bolívar et al., 2017). En la Cuenca Oriental, tanto el epilimnion como el agua subterránea, tienen diversos usos que incluyen: público-rural, agrícola y pecuario (Alcocer- Bernal-Brooks et al., 2019En el monitoreo que se realiza para evaluar la calidad del agua desde el punto vista microbiológico, es prácticamente imposible medir todos los organismos presentes; por ello se han desarrollado métodos en el que se consideran bacterias indicadoras (APHA, 2012). Así, el grupo de bacterias coliformes se aplica como prueba general de monitoreo de CMA y se ha utilizado a lo largo de los últimos 100 años para llevar a cabo estudios de agua potable, contaminación de sistemas acuáticos, fuentes de contaminación de aguas residuales crudas y sistemas de tratamiento de aguas residuales y aguas recreativas (APHA, 2012). También se ha propuesto a otros microorganismos para ser usados como indicadores alternativos o bioindicadores de calidad, e. g., enterococos, Escherichia coli, entre otros (Schoen et al., 2011; Bolívar et al., 2017), y más recientemente la resurrección de Clostridium perfringers como indicador de contaminación fecal antigua (Oh et al., 2021).

En la Meseta Central de México, se encuentra la Cuenca Oriental (5000 Km²), la cual es de tipo endorreica y es una de las más importantes del país (Alcocer- Bernal-Brooks et al., 2019); en ella, se localizan cuatro de los seis lagos cráter sódicos que existen para el estado de Puebla (Cruz-Aviña et al., 2017). En esta localidad, reciben el nombre de Axalapascos: Atexcac, Alchichica, La Preciosa y Quechulac (Alcocer- Bernal-Brooks et al., 2019). Estos embalses, albergan una biodiversidad única, con especies endémicas acuícolas y terrestres (Alcocer-Bernal et al., 2019).

Al borde de estos cuerpos de agua, existen poblaciones rurales que fluctúan entre los 200 - 500 habitantes (Alcocer- Bernal-Brooks et al., 2019) y en algunos de ellos ocurre descarga directa de agua residuales sin tratamiento (Alcocer- Bernal-Brooks et al., 2019), por el desbordamiento de sistemas sépticos defectuosos (Can-Chulim et al., 2014), por heces fecales directas del ganado caprino u otros animales de compañía (Cruz-Aviña et al., 2017), así como por la formación de aluviones, que, por la condición endorreica de estos Axalapascos, y su suelo cinerítico, facilitan su deflación (García y Portilla, 2010). También se tiene documentado que desde hace más de 20 años se instaló una empresa transnacional porcícola, que actualmente cuenta con 16 granjas de alto impacto ambiental por el uso de bioaerosoles (Hernández-Avilés et al., 2012).

La contaminación fecal de las aguas de los Axalapascos es un problema complejo, el cual no ha sido estudiado ni abordado de manera sistemática, y que incide en la salud humana local y del ecosistema mismo. Se ha registrado un aumento creciente de enfermedades directas por el agua 
(Cruz-Aviña et al., 2017; Alcocer- Bernal-Brooks et al., 2019), que a su vez se relaciona también con un crecimiento de las poblaciones ribereñas y por la presencia de granjas pecuarias circundantes a la zona (Alcocer- Bernal-Brooks et al., 2019; Stentiford et al., 2020).

Una de las consecuencias por esta contaminación fue registrada por Can-Chulim et al., (2014), quienes documentaron que en la periferia de estos lagos existen pozos profundos contaminados por coliformes. No obstante, en México existen pocos informes sobre la calidad microbiológica superficial del agua, en general, y en embalses naturales en particular (Alcocer- Bernal-Brooks et al., 2019). Estos limitados estudios se basan principalmente en indicadores de contaminación fecal, como coliformes totales, coliformes fecales y enterococos, y han registrado que la calidad del agua no es apta para el consumo humano, ni para actividades agropecuarias, ya que la carga microbiana es elevada y esta puede afectar a organismos que ahí se desarrollan (EPA, 2012; Stentiford et al., 2020).

Por lo anteriormente expuesto, el objetivo de esta investigación fue: valorar la calidad microbiológica del agua superficial o epilimnion de cuatro Axalapascos, Alchichica, Atexcac, La Preciosa y Quechulac, mediante la presencia de microorganismos indicadores de contaminación fecal, así como la determinación de algunos parámetros fisicoquímicos ( $\mathrm{pH}$, temperatura, salinidad y oxígeno disuelto), relacionados con las condiciones de crecimiento de los grupos bacterianos de interés.

\section{MATERIAL Y MÉTODO}

\subsection{Descripción área de estudio}

Los Axalapascos son lagos de tipo "Maars" que se encuentran entre los N 18 $48^{\circ}-19^{\circ} 43^{\prime}$ y W 9709'-98 $03^{\circ}$ ', a 2312 m s.n.m., en el Altiplano Mexicano, en los municipios de Guadalupe Victoria y Tepeyahualco, Puebla (Figura 1), donde prevalece un clima semiárido-templado (García, 2020). La colecta se llevó a cabo en 4 lagos cráter: Alchichica (ALCHI), Atexcac (ATx), la Preciosa (LPr) y Quecholac (QUE). La zona geográfica está catalogada como Región Hidrológica Prioritaria (RHP 070) y Región Terrestre Prioritaria (RTP 122) por la Comisión Nacional de la Biodiversidad de México (CONABIO, 2014).

\subsection{Sitios de muestreo microbiológico}

Para evaluar la presencia de contaminación microbiológica de los Axalapascos, se realizaron muestreos entre febrero del 2012 y febrero del 2013. Esto permitió dirimir las temporadas de secas y lluvias, así como los periodos de termoclina y mezcla (APHA, 2012). La toma de muestras se efectuó en 10 sitios por lago (incluido el centro donde se encuentra una boya de la UNAM que representa el sitio más profundo), de manera mensual y por triplicado (500 $\mathrm{mL}$ en cada toma).

Se elaboró un análisis de componentes principales con el programa $\mathrm{R}$ commander para determinar si había diferencias significativas entre los lugares de muestreo; al no encontrarse diferencias significativas se seleccionó un sitio de muestreo representativo para cada lago cráter (APHA, 2012), que fue georreferenciado con un GPS, Garmín XL12. 

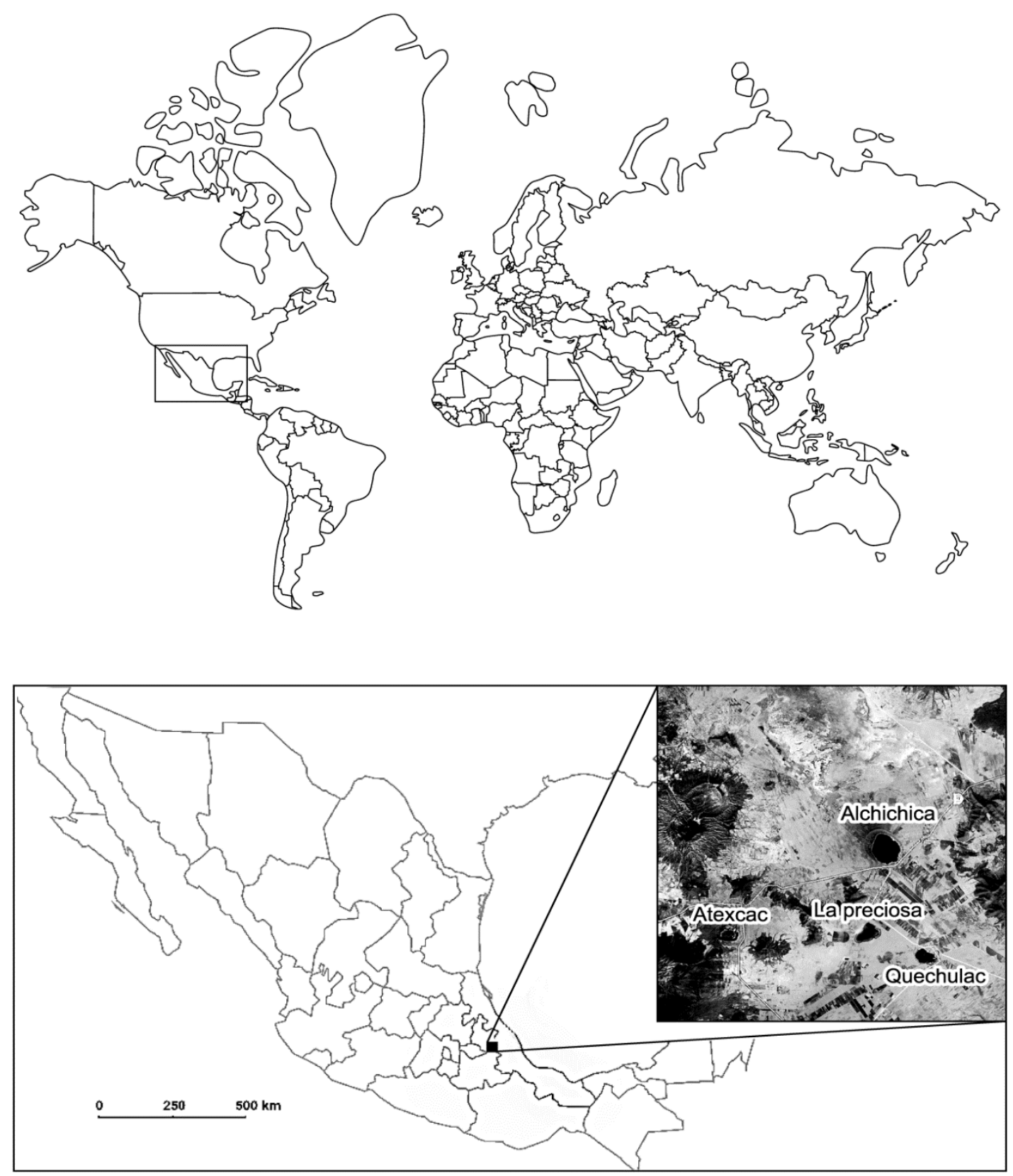

Figura 1. Localización geográfica de los lagos cráter, ALCHI = Alchichica; ATx = Atexcac; $\mathrm{LPr}=\mathrm{La}$ Preciosa; QUE $=$ Quechulac. N $18^{\circ} 48^{\prime}-19^{\circ} 43^{\prime}$ y W $97^{\circ} 09^{\prime}-98^{\circ} 03^{\prime}$, a 2312 m. s.n.m. Se ubican en los municipios de Guadalupe Victoria y Tepeyahualco en el estado de Puebla, México.

\subsubsection{Colecta y transporte de muestras}

Se utilizaron recipientes de vidrio estériles, por triplicado, que fueron transportados en hielo al laboratorio de patogenicidad bacteriana del Centro de Investigación en Ciencias Microbiológicas de la Benemérita Universidad Autónoma de Puebla, en un tiempo no mayor a 4 h y analizadas el mismo día de su muestreo (APHA, 2012). 


\subsubsection{Determinación de los parámetros fisicoquímicos}

Los parámetros fisicoquímicos: $\mathrm{pH}$, salinidad, oxígeno disuelto y temperatura, fueron analizados mensualmente in situ, a través de perfiles, mediante una sonda multiparamétrica Hydrolab modelo Datasonde (APHA, 2012).

2.3 Fase de laboratorio

La CMA se determinó a través de indicadores de calidad microbiológica conforme a los Métodos Standard para el Examen de Aguas y Aguas Residuales de la American Public Health Association (APHA, 2012) y las Normas Oficiales Mexicanas vigentes e internacionales (EPA, 2012).

\subsubsection{Cuantificación de enterococos (ENT)}

Se cuantificaron de acuerdo con SEMARNAT (1997) que establece los límites máximos permisibles (LMP) de calidad conforme a su uso y destino.

\subsubsection{Coliformes totales (CT)}

Se empleó la técnica del número más probable (NMP) y tubos de dilución múltiple de fermentación referidos en la NMX 042 AA. El método consistió en dos pruebas, una presuntiva y otra confirmatoria.

\subsubsection{Coliformes fecales (CF)}

Se realizaron los métodos descriptos en SSA (1994) y las recomendaciones de la SEMARNAT (1997) para calidad del agua y el NMP de CF (termotolerantes) y E. coli presuntiva. Adicionalmente, se realizó la comparación con los LMP para agua de consumo humano, pecuario y de esparcimiento dentro del marco de las (EPA, 2012).

\subsection{Análisis estadísticos}

Para obtener los diferentes análisis estadísticos se usó el paquete estadístico IBM SPSS versión 22. La determinación de diferencias significativas entre los parámetros microbiológicos y fisicoquímicos de los cuatro lagos cráter se efectuó mediante pruebas no paramétricas de rutina (Levene, Tukey y Análisis de Varianza - ANOVA -); adicionalmente se elaboraron las pruebas de correlación de Person y de análisis por componentes principales. Por otro lado, se realizó una matriz de covarianza para compensar la diferencia numérica entre las distintas variables y se efectuaron las pruebas de Kaiser, Meyer y Olkin (KMO) y de esfericidad de Pearson para conocer la idoneidad del análisis. Por último, se hizo un análisis del comportamiento entre las poblaciones de bacterias coliformes por Axalapasco, utilizando el método de modelo lineal general (univariante) y unidireccional.

\section{RESULTADOS}

3.1 Caracterización microbiológica del agua de los Axalapascos 


\subsubsection{Coliformes Totales}

Se detectaron en el epilimnion de los cuatro lagos cráter estudiados. Los lagos Alchichica y Quechulac presentaron la mayor carga bacteriana, con hasta $2500 \mathrm{NMP} 100 \mathrm{~mL}^{-1}$ para ambos lagos durante el mes de mayo. De hecho, el límite máximo permisible para agua de consumo humano es de 0-0.5 NMP $100 \mathrm{~mL}^{-1}$, el de riego, uso agrícola y pecuario de 2000-1000 NMP $100 \mathrm{~mL}^{-1}$ y el de actividades recreativas es de 4000-5000 NMP $100 \mathrm{~mL}^{-1}$, por lo que ambos lagos rebasan estos parámetros.

\subsubsection{Coliformes Fecales}

Se registraron en el epilimnion de Quechulac hasta $1400 \mathrm{NMP} 100 \mathrm{~mL}^{-1}$ para el mes de septiembre y con 1000-800 NMP $100 \mathrm{~mL}^{-1}$ para los meses de mayo, junio y octubre (Figura 2), valores que rebasaron la normatividad del orbe actual. El lago La Preciosa también presentó dos picos idénticos para los meses de junio y julio con $450 \mathrm{NMP} 100 \mathrm{~mL}^{-1}$. El lago menos contaminado por este grupo bacteriano fue Atexcac con promedios de 700-500 y 300-100 NMP $100 \mathrm{~mL}^{-1}$ durante la época de secas.

\subsubsection{Enterococos}

Se registraron en los 4 lagos. En los lagos Quechulac y Alchichica se observaron los valores promedio más altos $\left(\right.$ Quechulac $=2000 \mathrm{NMP} / 100 \mathrm{~mL}^{-1}$, Alchichica $\left.=1000 \mathrm{NMP} / 100 \mathrm{~mL}^{-1}\right)$. Al comparar estos resultados con los últimos datos disponibles de la Red Nacional de Monitoreo de la Comisión Nacional del Agua (2003-2013), y con la normatividad sanitaria vigente y sus proyectos de mejora ambiental, se observó una tendencia al incremento de enterococos principalmente en la época de estiaje. Así mismo se rebasó la norma internacional para este grupo, en todos los Axalapascos en al menos un mes evaluado, siendo en algunos casos los conteos bacterianos altos (2300 NMP/100 $\left.\mathrm{mL}^{-1}\right)$. Estos conteos también superaron lo estipulado por la norma oficial para agua de riego agrícola y protección de la vida acuática (2000-1000 NMP100 $\mathrm{mL}^{-1}$ ) (Figura 2).

\subsubsection{Escherichia Coli}

Estuvo presente en todos los Axalapascos, con valores de 1500 para Quechulac y de 1000 para Alchichica, que están por arriba de la normatividad vigente para aguas de uso agrícola, pecuario o recreativo. Conforme a la (12) se establece que el agua de estos embalses no debe presentar E. coli en ninguna de las muestras estudiadas por $100 \mathrm{~mL}^{-1}$. En la NOM se referencia que, en poblaciones de más de 50, 000 habitantes, estos organismos deberán estar ausentes en un $95 \%$ en las muestras tomadas en cualquier periodo de 2 meses, situación que no ocurre de acuerdo a nuestros datos. Esto se aprecia principalmente en el caso del lago Quechulac, que presentó el mayor conteo promedio y el pico más alto para la época de secas y lluvias (Figura 2). 
A

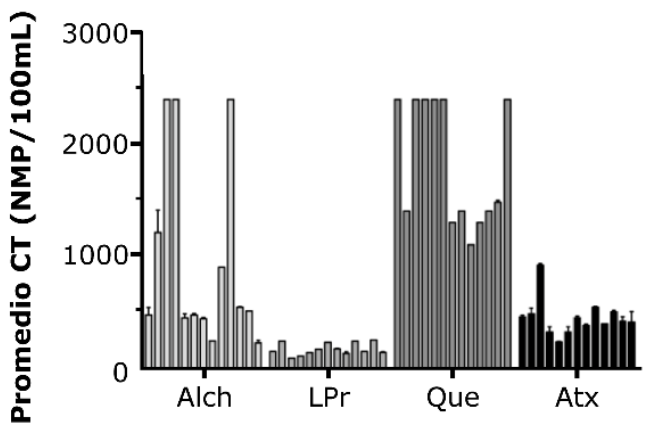

C

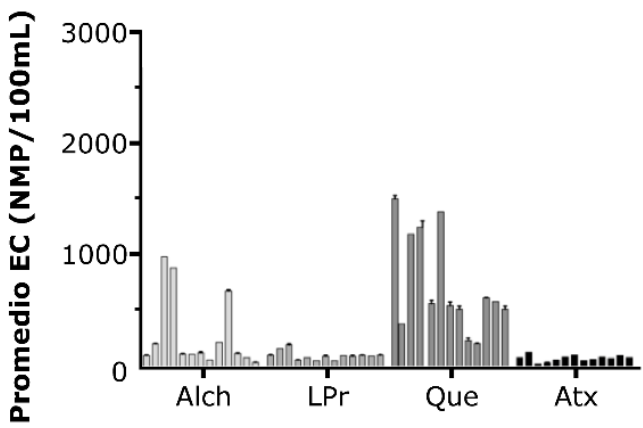

B

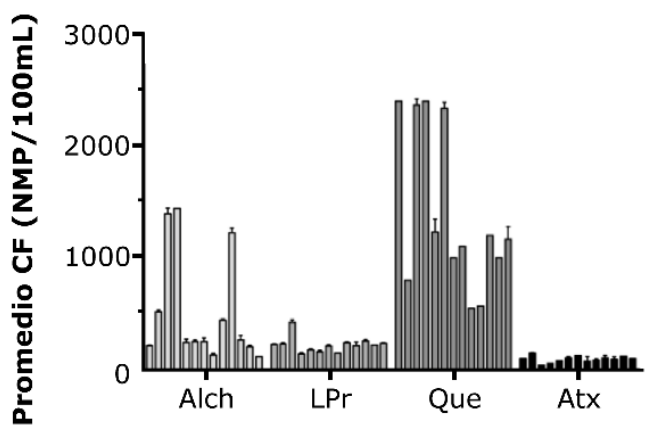

D

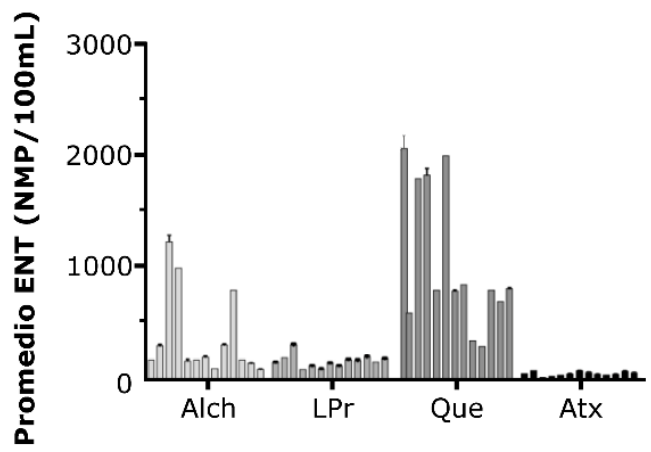

Figura 2. Conteos bacterianos, mediante la técnica del número más probable (NMP) de Coliformes Totales (CT), Coliformes Fecales (CF), E. coli (EC) y Enterococos (ENT) para cada lago cráter estudiado. ALCHI = Alchichica; $\mathrm{ATx}=$ Atexcac; $\mathrm{LPr}=$ La Preciosa; $\mathrm{QUE}=$ Quechulac.

\subsection{Parámetros Fisicoquímicos del agua de los Axalapascos}

Los cuatro Axalapascos presentaron un $\mathrm{pH}$ alcalino con poca variabilidad a lo largo del año, cercano a $9.0 \pm 0.5$, con un pico de 9.2 para Alchichica. La salinidad fue variable para cada lago cráter. Los lagos de Alchichica y Atexcac se definieron como atalasohalinos $\left(7-6 \mathrm{gL}^{-1}\right)$, mientras que el lago La Preciosa resultó subhalino (1.0-1.5 $\left.\mathrm{gL}^{-1}\right)$, y finalmente Quechulac fue hipohalino o dulce $\left(0.5-0.0 \mathrm{gL}^{-1}\right)$ (Figura 3). 
A

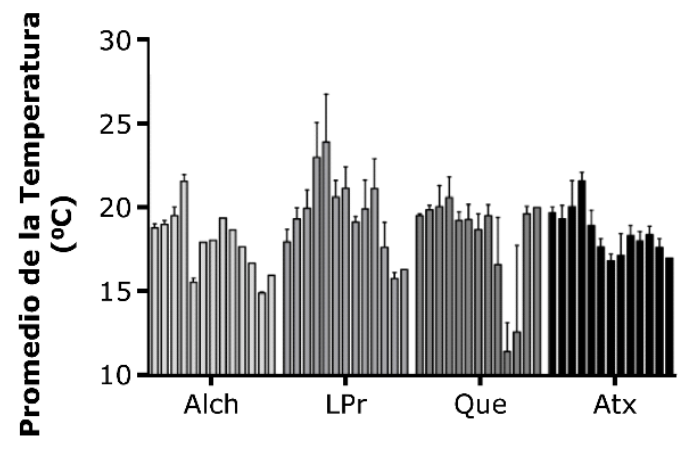

C

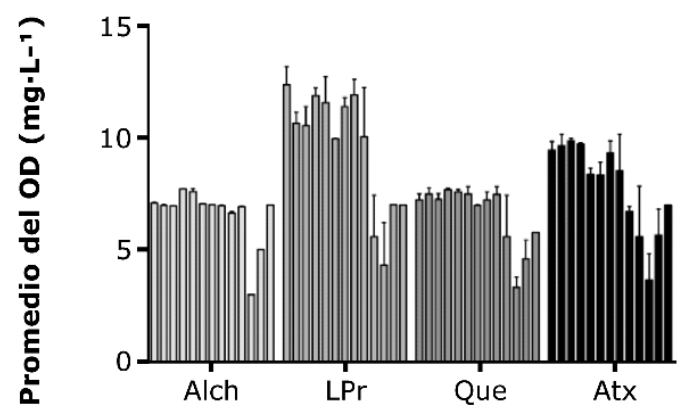

B

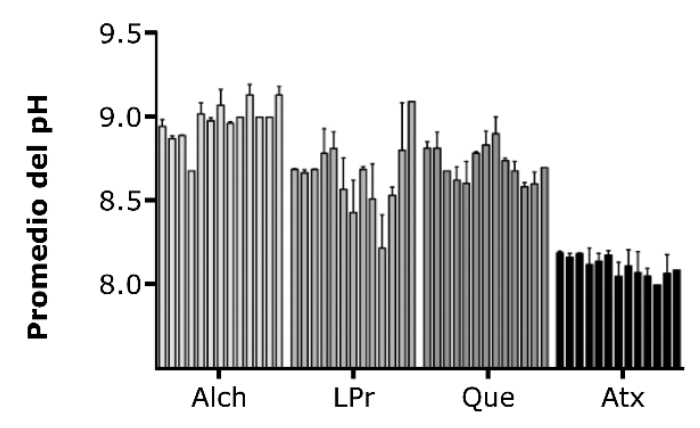

D

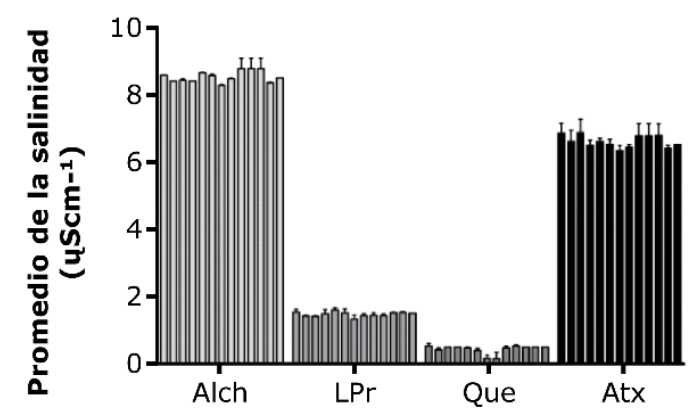

Figura 3. Parámetros fisicoquímicos (temperatura, $\mathrm{pH}$, Oxígeno disuelto y Salinidad) para cada lago cráter estudiado. ALCHI = Alchichica; ATx = Atexcac LPr = La Preciosa; QUE = Quechulac.

El porcentaje y saturación de oxígeno disuelto (OD) presentó poca variación durante la época de termoclina y solo cambió en verano llegando a la sobresaturación $\left(9-10 \mathrm{mgL}^{-1}\right)$, y en la época de circulación o mezcla, que abarca los meses de diciembre a marzo, donde hubo un abatimiento en la concentración de OD (hasta 2-3 mgL-1). La temperatura promedio varió en todos los casos de $20^{\circ}$ a $10^{\circ} \mathrm{C}$, siendo el menor registro en el lago de Atexcac con $7^{\circ} \mathrm{C}$ en la época de mezcla (Figura $3)$. En cuanto a los coliformes se tiene una relación entre el número más probable para coliformes totales, coliformes fecales y E. coli, obteniéndose tres picos de coliformes fecales que sobrepasan los $2000 \mathrm{NMP} / 100 \mathrm{~mL}$ en la figura 4A (ALCHI), siendo estos mismos números altos en la figura 4B (QUE), marcando todo lo contrario para la figura 4C y 4D, con números menores a 500 NMP/100mL (LPr y Atx). De igual forma en el último lago (ATx) no se reportó E. coli, ni enterococos, y el $\mathrm{pH}$ mantiene niveles por debajo de 8.5 para $\mathrm{C}$ y $\mathrm{D}$, entre febrero/2012 y febrero/2013 (Figura 4). 


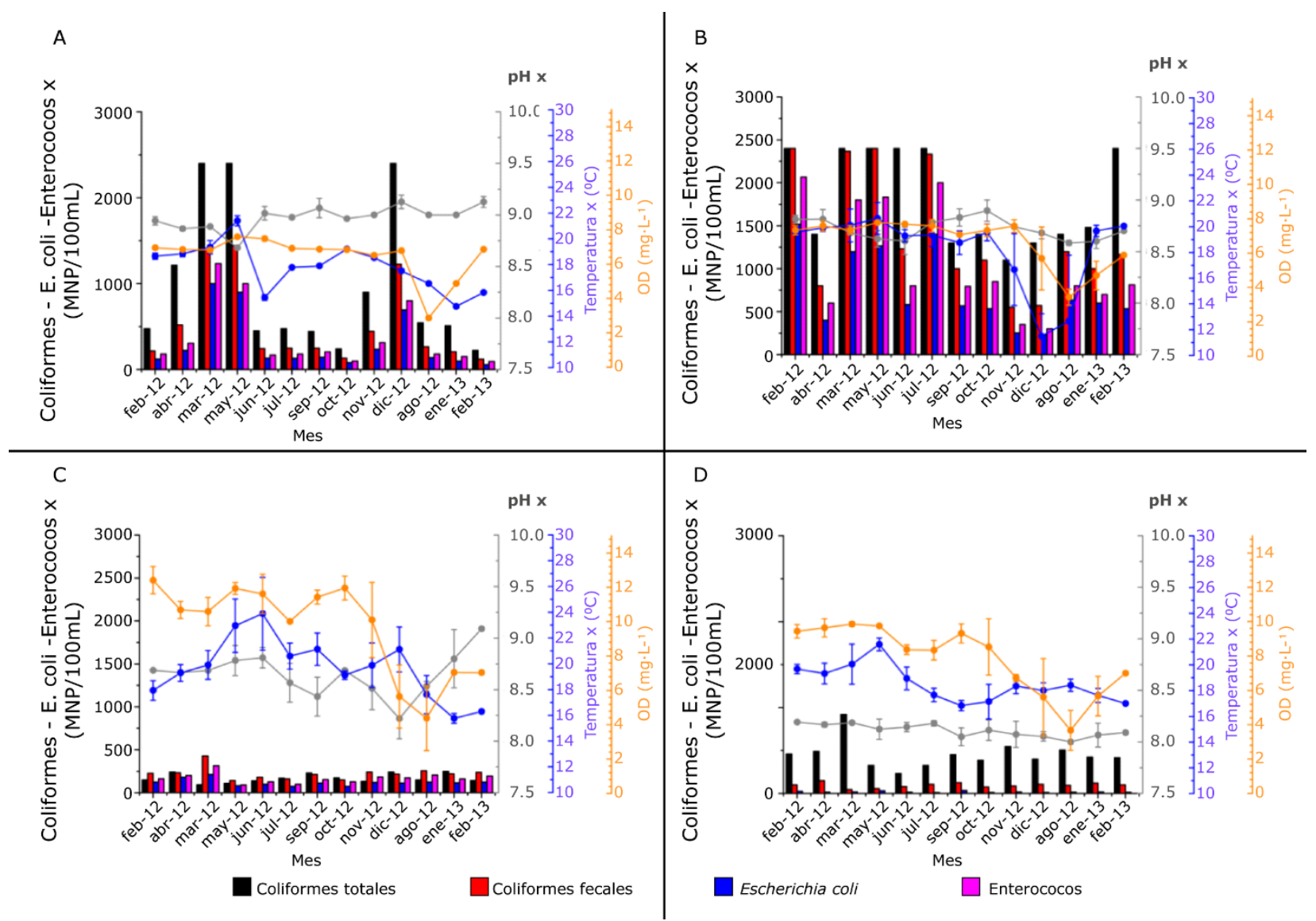

Figura 4. Relación entre el número más probable (NMP) para Coliformes Totales, Coliformes Fecales y E. coli, con los parámetros de Temperatura $\left(\mathrm{T}^{0} \mathrm{C}\right), \mathrm{pH}$, Salinidad $(\% 0)$ y Oxígeno Disuelto (OD mgL $\left.{ }^{-1}\right)$ en A) ALCHI, B) QUE, C) LPr y D) ATx, entre febrero/2012 y febrero/2013.

\subsection{Análisis estadísticos}

El análisis ANOVA indicó que existe diferenciación en las concentraciones totales de bacterias entre ALCHI-QUE, LPr-QUE ( $\mathrm{p}<0.05)$ y QUE-ATx $(\mathrm{p}<0.05)$. También se presentaron diferencias en la presencia del grupo coliforme-coliforme fecal entre ALCHI-QUE y QUE-ATx $(\mathrm{p}<0.05$, en ambos casos) (Tabla 1, Figura 5). En el recuento del grupo de los enterococos solamente QUEATx $(\mathrm{p}<0.05)$ y LPr-QUE mostraron diferencias significativas $(\mathrm{p}<0.05)$. 


\section{A}

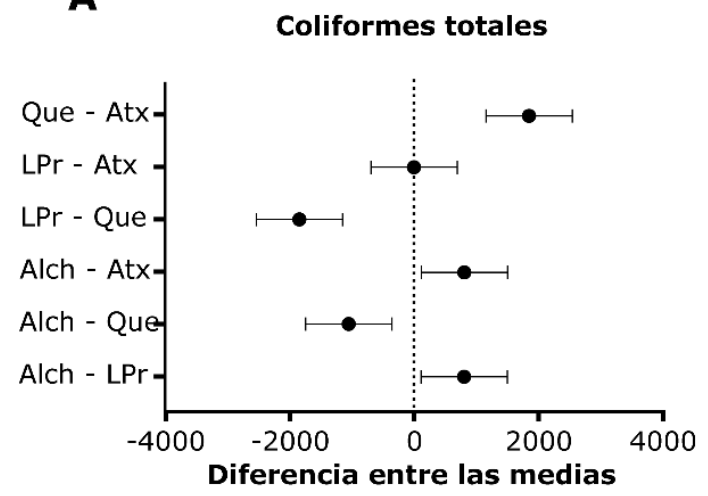

\section{C}

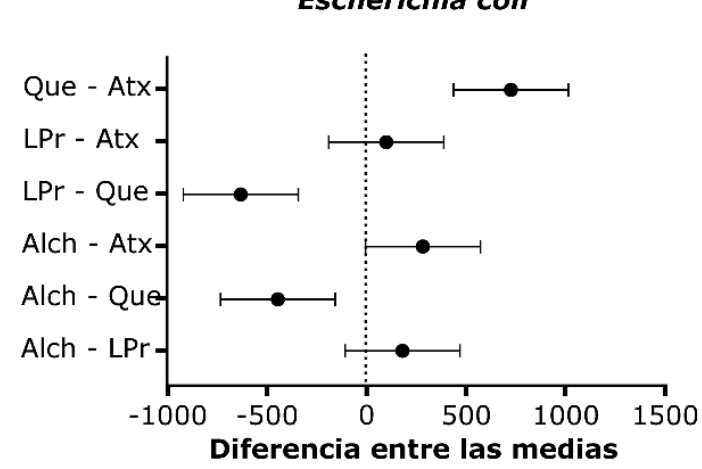

B

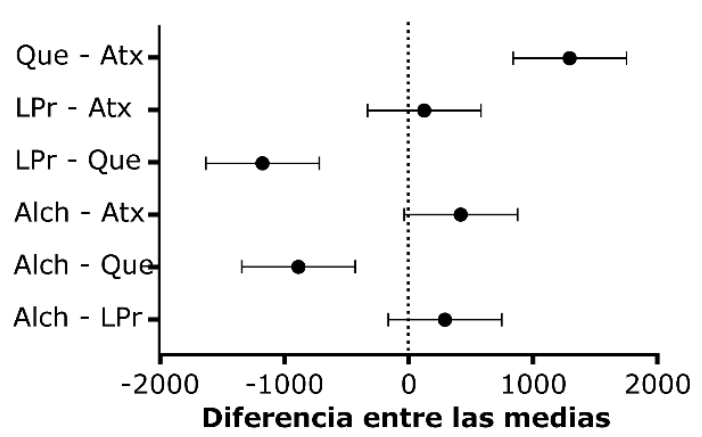

D

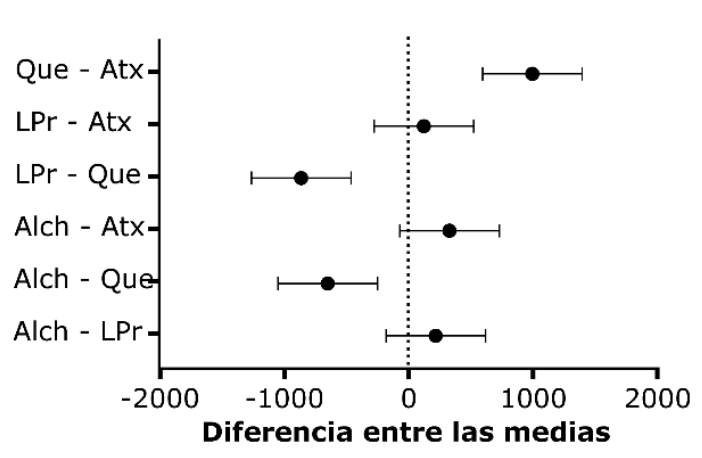

Figura 5. Intervalos de confianza (95\%) de la prueba de Tukey para los cuatro microorganismos indicadores determinados en los lagos cráter. ALCHI = Alchichica; ATx = Atexcac; LPr = La Preciosa; QUE = Quechulac.

Tabla 1. Resultados del análisis de correlación ANOVA en los cuatro lagos estudiados. $\overline{\mathrm{x}}=$ Media; $\sigma=$ Desviación estándar; CV $(\%)$ = Coeficiente de variación.

\begin{tabular}{clcccc}
\hline & & Alchichica & La Preciosa & Quechulac & Atexcac \\
\hline \multirow{3}{*}{ Coliformes totales } & $\overline{\mathrm{x}}$ & 975.2 & 171 & 2014 & 171 \\
& $\sigma$ & 852.3 & 52.51 & 990.7 & 52.51 \\
& $\mathbf{C V}(\boldsymbol{\%})$ & $\mathbf{8 7 . 3 9}$ & $\mathbf{3 0 . 7 1}$ & $\mathbf{4 9 . 1 9}$ & $\mathbf{3 0 . 7 1}$ \\
& $\overline{\mathrm{x}}$ & 516.1 & 223.2 & 1394 & 95.31 \\
Coliformes fecales & $\sigma$ & 491.3 & 71.22 & 713.5 & 28.19 \\
& $\mathbf{C V}(\boldsymbol{\%})$ & $\mathbf{9 5 . 1 9}$ & $\mathbf{3 1 . 9}$ & $\mathbf{5 1 . 1 9}$ & $\mathbf{2 9 . 5 8}$ \\
& $\overline{\mathrm{x}}$ & 304.5 & 119.8 & 747.1 & 18.41 \\
& $\sigma$ & 329.2 & 39.14 & 439.3 & 7.564 \\
& $\mathbf{C V}(\boldsymbol{\%})$ & $\mathbf{1 0 8 . 1 2}$ & $\mathbf{3 2 . 6 7}$ & $\mathbf{5 8 . 7 9}$ & $\mathbf{4 1 . 0 8}$ \\
& $\overline{\mathrm{x}}$ & 378.1 & 169.6 & 1039 & 58.1 \\
& $\sigma$ & 377 & 56.89 & 640.2 & 25.41 \\
& $\mathbf{C V}(\boldsymbol{\%})$ & $\mathbf{9 9 . 7 2}$ & $\mathbf{3 3 . 5 5}$ & $\mathbf{6 1 . 6 1}$ & $\mathbf{4 3 . 7 4}$ \\
\hline
\end{tabular}




\begin{tabular}{clrrrr} 
& & Alchichica & La Preciosa & Quechulac & Atexcac \\
& $\overline{\mathbf{x}}$ & 18.49 & 19.66 & 18.22 & 17.97 \\
Temperatura & $\sigma$ & 1.379 & 2.384 & 2.922 & 1.829 \\
& $\mathbf{C V}(\boldsymbol{\%})$ & $\mathbf{7 . 4 6}$ & $\mathbf{1 2 . 1 3}$ & $\mathbf{1 6 . 0 4}$ & $\mathbf{1 0 . 1 8}$ \\
& $\overline{\mathrm{x}}$ & 8.111 & 8.652 & 8.719 & 8.973 \\
$\mathrm{pH}$ & $\sigma$ & 0.06098 & 0.2111 & 0.1019 & 0.1175 \\
& $\mathbf{C V}(\boldsymbol{\%})$ & $\mathbf{0 . 7 5}$ & $\mathbf{2 . 4 4}$ & $\mathbf{1 . 1 7}$ & $\mathbf{1 . 3 1}$ \\
& $\overline{\mathrm{x}}$ & 7.84 & 9.558 & 6.6 & 6.611 \\
Oxígeno Disuelto & $\sigma$ & 1.955 & 2.659 & 1.361 & 1.26 \\
& $\mathbf{C V}(\boldsymbol{\%})$ & $\mathbf{2 4 . 9 4}$ & $\mathbf{2 7 . 8 2}$ & $\mathbf{2 0 . 6 2}$ & $\mathbf{1 9 . 0 5}$ \\
& $\overline{\mathrm{x}}$ & 6.608 & 1.472 & 0.4391 & 8.536 \\
Salinidad & $\sigma$ & 0.184 & 0.07196 & 0.1273 & 0.169 \\
& $\mathbf{C V}(\boldsymbol{\%})$ & $\mathbf{2 . 7 8}$ & $\mathbf{4 . 8 9}$ & $\mathbf{2 9 . 0 0}$ & $\mathbf{1 . 9 8}$ \\
\hline
\end{tabular}

El análisis de Pearson no indicó correlación entre las variables microbiológicas y físico-químicas. Mientras que en el análisis por componentes principales se observa que los Coliformes Totales y Coliformes Fecales son discriminados por la influencia del componente 3, que aporta una variabilidad del $13.9 \%$ al sistema. Escherichia coli se mantiene alejado del clúster en dónde se agrupan las Coliformes Totales y los Enterococos. A pesar de que los Enterococos se encuentran escasamente resueltos por el componente 1, con respecto a las Coliformes Totales (Figura 6).

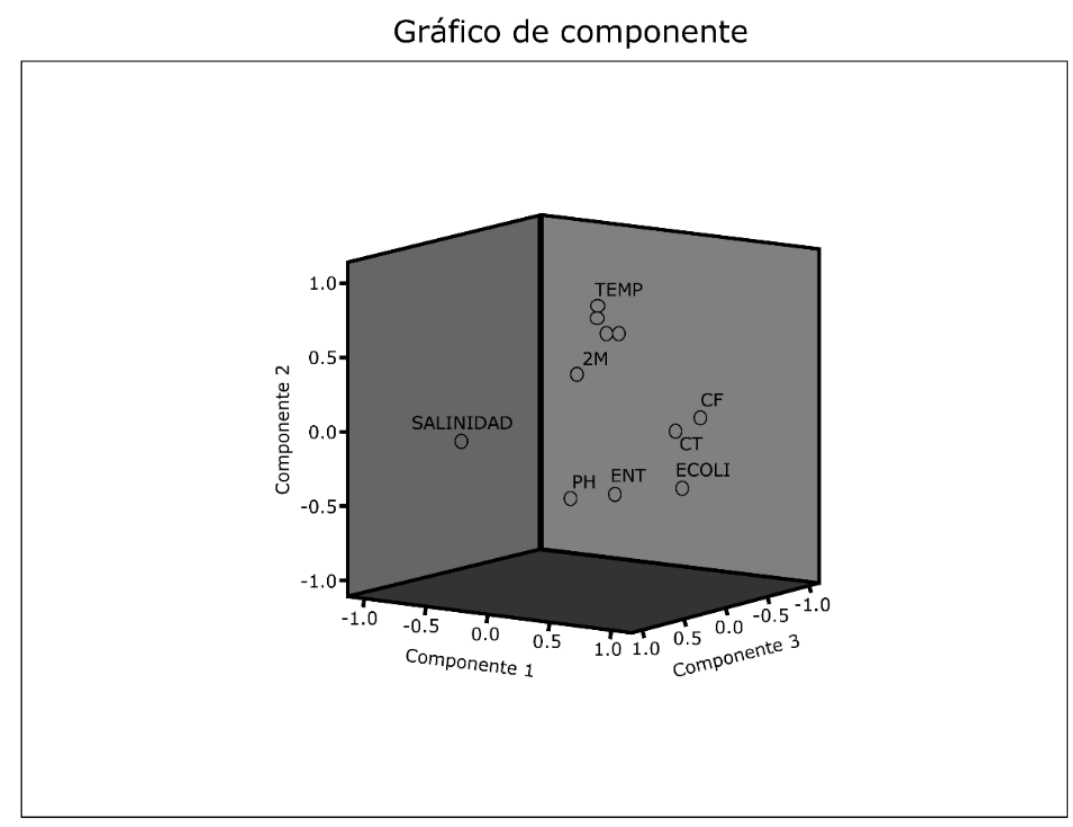

Figura 6. Gráfico en cubo derivado del Análisis de componentes principales de los datos fisicoquímicos del agua $v s$ datos microbiológicos. El componente 1 abarca el $31.7 \%$ del sistema; el componente 2, el $22 \%$ formando un clúster con los parámetros de salinidad, oxígeno disuelto y temperatura; el componente 3 agrupa a las coliformes totales, 
coliformes fecales y enterococos, con relación con el pH de los cuatro lagos cráter de este estudio con un $46 \%$ del sistema.

La cercanía entre los enterococos y el $\mathrm{pH}$ se explica por el componente 2 representado por $E$. coli con el $22 \%$ de la variabilidad del sistema, lo que sugiere que las variaciones de $\mathrm{pH}$ en el agua de los cuatro Axalapascos influyen más que otra variable en las fluctuaciones de estas bacterias indicadoras (Figura 6).

La temperatura del agua y el oxígeno disuelto se encuentran débilmente resueltos entre los componentes 1 y 2 , pero fuertemente resueltos por la influencia del componente 3 . Ambas variables están formando un clúster, sugiriendo que las fluctuaciones entre ambas están relacionadas. Por último, la salinidad es una variable que se encuentra mayormente resuelta por la influencia del componente 1 por lo que su influencia global a las fluctuaciones de otras variables no se puede establecer (Figura 6).

\section{DISCUSION}

Los resultados de este trabajo indicaron la presencia de enterococos, coliformes totales, coliformes fecales y $E$. coli en los lagos cráter estudiados durante todo un año (época de secas y lluvias). Estos organismos son miembros importantes de las comunidades del intestino de muchos animales y el humano (Santiago-Rodríguez et al., 2010), y específicamente los enterococos son microorganismos patógenos oportunistas que causan múltiples infecciones (Schoen et al., 2011), por lo que actualmente son utilizados como indicadores de contaminación fecal (Lew et al., 2019). Los enterococos son resistentes a los cambios de temperatura corporal, cuando salen del intestino de los animales o humano y contaminan el agua (Santiago-Rodríguez, et al., 2010; Schoen et al., 2011), además se adaptan a temperaturas más bajas como las de los lagos cráter, que tienen en promedio $18^{\circ} \mathrm{C}$ (Lew et al., 2019); también son salino tolerantes (Santiago-Rodríguez et al., 2010), por su capacidad de sobrevivir a concentraciones variables de $\mathrm{NaCl}$ (Lew et al., 2019).

Los valores de enterococos, coliformes totales, coliformes fecales y E. coli fueron altos (Figura 2), sobre todo en temporada de lluvias; inclusive rebasaron los valores máximos de la normatividad vigente (SSA, 1994; Stentiford et al., 2020) para agua de uso recreacional, agrícola y pecuario (Santiago-Rodríguez et al., 2010), y similares a los reportados en un estudio para Pátzcuaro, Zirahuén, Xochimilco y Metztitlán, que actualmente se consideran como embalses con un riesgo sanitario alto. Destaca el hecho de que sobrepasan los índices establecidos en la normatividad (Santiago-Rodríguez et al., 2010), a pesar de que los Axalapascos estudiados son de tipo endorreico, con un régimen hidrológico negativo, y de no estar cerca de grandes ciudades y sus descargas. En los meses de noviembre, diciembre y enero, que corresponde a los meses de la temporada de secas y al periodo de mezcla en los lagos de QUE, ALCHI y ATx (Alcocer- BernalBrooks et al., 2019), los resultados de este trabajo indicaron una disminución de las poblaciones de enterococos.

Las fuentes de contaminación de los lagos La Preciosa y Atexcac son desconocidos, pero existe la probabilidad de que sean contaminados, por un lado, directamente por las comunidades que se encuentran en sus riberas (Alcocer- Bernal-Brooks et al., 2019) y, por otro lado, por las descargas de materia fecal, debido al pastoreo del ganado y animales de compañía en el borde de estos lagos 
(CONEVAL, 2020). Se recomienda la elaboración de estudios que precisen y comprueben las fuentes exactas del origen de la contaminación en estos lagos.

En este trabajo se comprobó que los ecosistemas acuáticos cerrados tipo lago cráter o Axalapascos, son sistemas frágiles debido a sus características geomorfológicas (tipo de suelo, pendiente, permeabilidad, etc.) y por ende susceptibles a los impactos rápidos de contaminación, incluida la microbiológica (Hernández-Avilés et al., 2012). En este sentido, García y Portilla (2010) describieron un agua casi prístina en el lago QUE, no obstante, en nuestro estudio la presencia de E. coli fue notoria (hasta $5000 \mathrm{NMP}$ o su equivalente en $1300 \mathrm{UFC} 100 \mathrm{~mL}^{-1}$ ). Cruz-Aviña et al. (2017) también probaron la sobrevivencia de Brucella spp. en el agua de los lagos cráter QUE, ALCHI, ATx y LPr y lo atribuyeron al resultado de la influencia antropogénica, derivada de malas prácticas agropecuarias (ganadería extensiva, trashumancia), ejercidas por los pobladores de la Cuenca Oriental por más de 95 años.

Cabe señalar que en el lago de QUE se observaron diversos animales (cerdos, perros, cabras, caballos, burros, vacas, entre otros) dentro del agua, así como personas nadando y pescando durante los meses del estudio, eventos que posiblemente favorecieron la presencia de estas bacterias. También se detectaron efluentes de aguas residuales, provenientes de casas de los poblados de las dos comunidades aledañas a Quechulac, Santa Cruz y Xaltepec. Adicionalmente, a una distancia menor de $4 \mathrm{Km}$, se tiene documentada la presencia de granjas de cerdos que constan de un pozo profundo y lagunas de oxidación al aire libre, con la formación de bioaerosoles (Hernández-Avilés, 2012). Hasta el momento no se ha comprobado su impacto directo en el lago, pero sería importante dirigir estudios que respondan el posible impacto que pudieran generar estas granjas en la calidad del agua del lago QUE.

Destaca el hecho de que los valores registrados de CT, CF, E. coli y ENT no presentaron diferencias significativas al compararlas con el oxígeno disuelto, la temperatura y la salinidad; en contraste, al compararlas con los valores de $\mathrm{pH}$, la asociación resultó significativa. Esto se puede atribuir a que estos organismos se caracterizan por ser anaerobios facultativos (Byappanahalli et al., 2012). En general, el pH del epilimnion de los lagos resultó alcalino, congruente a lo reportado por Lew et al., (2019) para varios lagos contaminados en E.U. El lago más alcalino fue Alchichica con valores máximos de $\mathrm{pH}$ de 9.2, mientras que el lago menos alcalino fue ATx con valores mínimos de 8.2. El lago LPr presentó variaciones de $\mathrm{pH}$ en los meses de junio a noviembre, alcalinizándose en los meses de diciembre, enero y febrero, que de acuerdo a APHA (2012) corresponden al periodo de secas. En este mismo periodo, se registró que cuando los lagos presentaron una mayor alcalinidad en el $\mathrm{pH}$, las poblaciones microbianas decrecieron notablemente.

Los cambios en los valores de $\mathrm{pH}$ en los lagos de La Preciosa (8.5-8.7) y Quechulac (8.0-8.5) se dieron en la etapa de lluvias, que se acompaña de una disminución en la salinidad debido a que durante este periodo las escorrentías de las aguas se llevan residuos en suspensión como sales húmicas, minerales, partículas inorgánicas, vegetales en descomposición, y que al oxidarse inducen cambios de color y de $\mathrm{pH}$ (Byappanahalli et al., 2017). En la época de lluvias pueden inclusive presentarse efectos de alcalinización natural vía disolución de rocas y minerales de metales alcalinos y alcalinos térreos de la composición de las orillas de los lagos (Hernández-Avilés et al., 2012). A su vez pueden incrementar las concentraciones de microorganismos y el contenido de bacterias en el agua (Flohr et al., 2020), lo cual podría explicar las altas concentraciones de 
contaminantes fecales en épocas de lluvias, como ha sido comprobado en otros sistemas fluviales (Ávila de Navia et al., 2014).

Considerando los resultados de contaminación bacteriana de este trabajo es importante que las instancias competentes diseñen estrategias para la preservación de los lagos cráter estudiados, que tienen amplia relevancia económica y biológica en el estado de Puebla, lo que a su vez conllevará a la protección de la salud de las poblaciones locales y turísticas.

\section{CONCLUSION}

En este estudio se demostró la presencia de enterococos, coliformes totales, coliformes fecales y E. coli en los cuatro lagos cráter analizados durante todo un año, sugiriendo que estos lagos están contaminándose por bacterias de origen fecal derivadas de humanos y animales domésticos. Las temporadas de lluvias y secas en el ciclo analizado 2012-2013, demuestran ser una marcada influencia para las variaciones microbianas de cada lago cráter, siendo el lago cráter QUE el más contaminado en todos los aspectos durante este estudio y en contraste el lago ATx el de menor riesgo. Por su parte, los valores del pH en la capa superficial o epilimnion de los cuatro Axalapascos fueron alcalinos y fue el factor fisicoquímico determinante, y que más impactó a las poblaciones microbianas de este estudio, mientras que los otros parámetros: la temperatura, el oxígeno disuelto y la salinidad, no fueron determinantes ni estadísticamente significativos. La salinidad fue un parámetro variable para cada Axalapasco donde el agua de QUE fue de tipo hipohalina, LPr fue subhalina, en contraparte para ATx y ALCHI que fue mesohalina. Los hallazgos de este trabajo son relevantes y podrían servir de base para otros estudios de caso debido a la importancia biológica, ecológica y ambiental que representan estos lagos cráter.

\section{AGRADECIMIENTOS}

El primer autor desea agradecer a los Drs. Javier Alcocer, Miroslav Macek Jorge Ciros por el apoyo académico y económico brindado. Un agradecimiento especial al joven Ricardo Daniel Cruz Guerrero por todo su apoyo en campo. A Doña Lety, Don Juan y Don Lupe, habitantes de la comunidad de San Luis Atexcac, por su hospitalidad, atenciones y albergue.

\section{REFERENCIAS}

Alcocer, J., Bernal-Brooks, F.W. (2019). Physical and Chemical Characterization of Inland Waters. En: Ibáñez A. (Eds) Mexican Aquatic Environments, Springer, Cham. https://doi.org/10.1007/978-3-03011126-7-1.

APHA, AWWA WEF. (2012). Standard Methods for examination of water and wastewater. 22nd Ed. Washington: American Public Health Association, 1360 pp. ISBN 978-087553-013-0 http://www.standardmethods.org/.

Ávila de Navia, S.L., Estupiñán-Torres, S.M., Mejía Grajales, Á.M. y Mora Velásquez, L.V. (2014). La calidad bacteriológica del agua del humedal Jaboque (Bogotá, Colombia) en dos épocas contrastantes. Caldasia, 36 (2), 323-329. https:// doi: http://dx.doi.org/10.15446/caldasia/v36n2.4749. 
Bolívar, G.M., Pitre, I.A.J. y Correa, D.A. (2017). Cuantificación de coliformes totales en estuario del río ranchería. Biotecnología en el Sector Agropecuario y Agroindustrial, 15 (2): 23-29. https://doi.org/10.18684 / bsaa (15). 590.

Byappanahalli, M.N., Nevers, M.B., Korajkic, A., Staley, Z.R. y Harwood, V.J. (2012). Enterococci in the environment. Microbiology and Molecular Biology Reviews, 76(4):685-706. https://doi.org/10.1128/MMBR.00023-12.

Can-Chulim Á., Ortega-Escobar, H.M., Sánchez-Bernal, E.I. y Cruz-Crespo, E. (2014). Calidad del agua para riego en la Sierra Norte de Puebla, México. Tecnología y ciencias del agua, 5(5), 77-96. http://www.scielo.org.mx/scielo.php?pid=S2007-24222014000500005\&script=sci arttext.

CONABIO (Comisión Nacional para la Biodiversidad). (2014). Regiones Prioritarias Terrestres. RPT 122. Pico de Orizaba Cofre de Perote. México, pp. 475-479.

CONEVAL (Consejo Nacional de Evaluación de la Política de Desarrollo Social). (2020). Informe anual sobre la situación de pobreza y rezago social, Municipio de Guadalupe Victoria, Puebla, México. https://www.gob.mx/cms/uploads/attachment/file/38232/Puebla 067.pdf.

Cruz-Aviña, J.R., Castañeda-Roldán, E.I. y Silva-Gómez, S.E. (2017). La problemática ambiental de la región de los Axalapascos de Puebla: Erosión, pobreza, enfermedades, biodiversidad, etnocultura. En: Rodríguez HAL (Ed) El Desarrollo Sustentable, Plaza y Valdez, 1-99 pp.

EPA (Environmental Protection Agency United States). (2012). Guidelines for Water Reuse. EPA/600/R12/618, 145 pp. https://cedb.asce.org/CEDBsearch/record.jsp?dockey=0071833.

Flohr, B.M., Hunt, J.R., Kirkegaard, J.A., Rheinheimer, B., Swan, T., Goward, L. y Bullock, M. (2020). Deep soil water-use determines the yield benefit of long-cycle wheat. Frontiers in Plant Science, 11, 548. https://doi.org/10.3389/fpls.2020.00548.

García, E. (2020). Modificaciones al Régimen de Clasificación Climática de Köppen, México. http://www.igeograf.unam.mx/sigg/utilidades/docs/pdfs/publicaciones/geo_siglo21/serie_lib/modific_al_s is.pdf. (consultado: 25 de febrero de 2020).

García, G.G., Portilla, S.J.S. (2010). Evaluación de la calidad microbiológica del Lago Cráter Quechulac, Puebla. Tesis de Licenciatura. Biología. FESI-UNAM, México, 71 pp.

Hernández-Avilés, J.S., Bertoni, R., Macek, M. y Callieri, C. (2012). Why bacteria are smaller in the epilimnion than in the hypolimnion? A hypothesis comparing temperate and tropical lakes. Journal of Limnology, 71 (1), 10-15. https://doi.org/10.4081/jlimnol.2012.e10.

Lew, S., Glińska-Lewczuk, K. y Lew, M. (2019). The effects of environmental parameters on the microbial activity in peat-bog lakes. PloS one, 14 (10), e0224441. https://doi.org/10.1371/journal.pone.0224441.

Oh, E.J., Kim, J.M. y Kim, J.K. (2021). Interrelationship between climatic factors and incidence of FBD caused by Clostridioides difficile toxin B, Clostridium perfringens, Campylobacter spp., and Escherichia coli O157: H7. Environmental Science and Pollution Research, 1-9. https://doi: 10.1007/s11356-02113854-1. 
Sánchez-Alonso, R. E., González-Barbosa, J. J., Castillo-Castañeda, E. y García Murillo, M. A. (2016). Análisis cinemático de un novedoso robot paralelo reconfigurable. Revista Iberoamericana de Automática e Informática Industrial RIAI, 13 (2), 247-257.

Santiago-Rodríguez, T.M., Dávila, C., González, J., Bonilla, N., Marcos, P., Urdaneta, M. y Toranzos, G.A. (2010). Characterization of Enterococcus faecalis-infecting phages (enterophages) as markers of human fecal pollution in recreational waters. Water Research, 44, (1), 4716-4725. https://doi.org/10.1016/j.watres.2010.07.078.

Schoen, M.E., Soller, J.A. y Ashbolt, N.J. (2011). Evaluating the importance of faecal sources in humanimpacted waters. Water Research, 45 (8): 2670-2680. https://doi: 10.1016/ j.watres.2011.02.025.

SEMARNAT (Secretaría de Medio Ambiente y Recursos Naturales). (1997). Norma oficial mexicana NOM-003-SEMARNAT-1997. Que establece los límites máximos permisibles de contaminantes para las aguas residuales tratadas que se resumen en servicios al público. Secretaría de Medio Ambiente y Recursos Naturales. Diario Oficial de la Federación, Ciudad de México.

Simaan, N., Shoham, M. (2003). Stiffness synthesis of a variable geometry six-degrees-of- freedom double planar parallel robot. The International Journal of Robotics Research, 22 (9), 757-775.

SSA (Secretaría de Salud). (1994). NOM-022-SSA2-1994. Para la prevención y control de la brucelosis en el hombre, en el primer nivel de atención. Publicada en el Diario Oficial de la Federación, 30 de noviembre de $1995,55 \mathrm{pp}$.

Stentiford, G.D., Bateman, I.J., Hinchliffe, S.J., Bass, D., Hartnell, R., Santos, E.M. y Tyler, C.R. (2020). Sustainable aquaculture through the One Health lens. Nature Food, 1(8), 468-474. https://www.nature.com/articles/s43016-020-0127-5.

\section{SEMBLANZA DE LOS AUTORES}

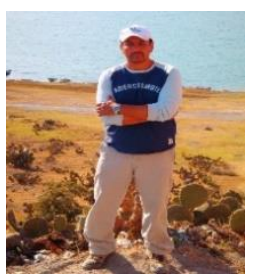

Juan Ricardo Cruz Aviña: Hidrobiólogo de profesión y Doctor en Medio Ambiente y Ciencias de la Salud por la BUAP. Sus trabajos fundamentales son en medio ambiente con énfasis en la conservación de anfibios, reptiles y peces autóctonos. Otros temas de interés han sido el desarrollo sustentable y educación ambiental.

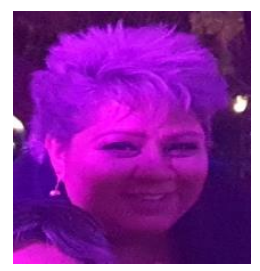

Laura G. Núñez-García: Hidrobióloga y Maestra en Biología por la Universidad Autónoma Metropolitana, México, donde actualmente como responsable Operativo de la "Planta Experimental de Producción Acuícola" en el Departamento de Hidrobiología. Ha desarrollado investigaciones dentro del campo de la acuicultura, ictiología y herpetología.

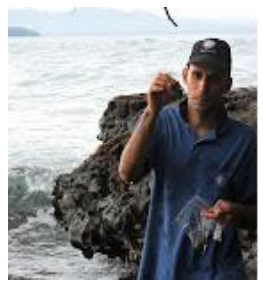

Ruben Cabrera: Biólogo de formación por la Universidad de La Habana. Ha desarrollado su investigación en osteología de peces, mamíferos y reptiles autóctonos en contextos arqueológicos. También tiene experiencia en ecología, sistemática de algas marinas. 


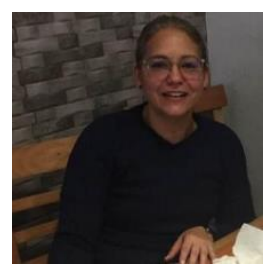

Jhoana Díaz-Larrea: Doctora en Ciencias Biológicas por la Universidad Autónoma Metropolitana, México. Profesor-Investigador Titular del Departamento de Hidrobiología, UAMI. Especialista en sistemática filogenética y biología molecular.

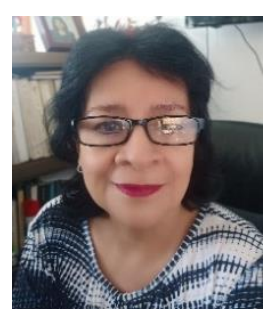

Elsa I. Castañeda-Roldán: Química Farmacobióloga por la Universidad Autónoma de Puebla, México, donde actualmente es profesor titular e investigadora. Trabaja líneas de investigación sobre medio ambiente y salud enfocadas a bacterias del género Brucella y ha desarrollado un medio de cultivo para el crecimiento rápido de estas bacterias patógenas, el que se distribuye a nivel nacional en México 